\title{
RECUPERAÇÃO DE OURO DE RESÍDUOS ELETRÔNICOS UTILIZANDO LIXIVIAÇÃO E BIOSSORÇÃO COM QUITINA
}

\author{
L. N. CORTÊS ${ }^{1}$, D. S. P. FRANCO ${ }^{1}$, E. H. TANABE ${ }^{1}$, D. A. BERTUOL ${ }^{1}$, G. L. DOTTO ${ }^{1 *}$ \\ ${ }^{1}$ Universidade Federal de Santa Maria, Departamento de Engenharia Química \\ *e-mail: guilherme_dotto@yahoo.com.br
}

\section{RESUMO}

Este trabalho visou à recuperação de ouro a partir de microprocessadores de computador descartados (MCD) utilizando lixiviação seguida de biossorção com quitina. Os componentes dos MCD foram lixiviados usando soluções de tioureia e, duas rotas foram testadas para recuperar o ouro a partir destas soluções: (1) biossorção e (2) precipitação seguida por biossorção. Para cada uma das rotas, a biossorção foi avaliada de acordo com a cinética e o equilíbrio. Para ambas as rotas, o modelo de ordem geral foi adequado para representar o comportamento cinético e, o equilíbrio foi bem representado pelo modelo de BET. Verificou-se que a precipitação seguida por biossorção foi mais eficiente para a recuperação de ouro, uma vez que os outros compostos foram removidos da solução lixiviada na etapa de precipitação. Usando esta rota, cerca de $80 \%$ do ouro foi recuperado, utilizando $20 \mathrm{~g} \mathrm{~L}^{-1}$ de quitina a $298 \mathrm{~K}$ durante $4 \mathrm{~h}$.

\section{INTRODUÇÃO}

A indústria eletrônica tornou-se um dos setores que mais crescem no mundo. Como consequência, grandes quantidades de resíduos eletrônicos (RE) são geradas (Wang e Xu, 2014). Demonstrou-se que mais de um bilhão de computadores e seus microprocessadores foram descartados entre 1997 e 2010, apenas no Japão e nos Estados Unidos (Kiddee et al., 2013). O tratamento incorreto dos RE é um grave problema de poluição, uma vez que contém uma variedade de substâncias tóxicas, as quais podem contaminar o meio ambiente e ameaçar a saúde humana (Hong et al., 2015). Em paralelo, os RE contém metais valiosos, tais como, ouro, prata, platina e cobalto, que podem ser recuperados (Bertuol et al., 2015). Desta forma, o desenvolvimento de tecnologias para recuperação de metais de RE é uma alternativa para obter um produto valioso e resolver um problema ambiental (Vidyadhar et al., 2013).
A biossorção é uma tecnologia promissora para a remoção de metais pesados a partir de soluções aquosas. As principais vantagens da biossorção são a sua elevada eficácia na redução dos íons de metais pesados e a utilização de biossorventes baratos. Operações de biossorção são particularmente adequadas para o tratamento de soluções diluídas de metais pesados (Ungureanu et al., 2015). No entanto, não existem estudos utilizando quitina como biossorvente para a recuperação de ouro.

A quitina é o segundo polissacarídeo natural mais abundante e o principal componente estrutural do exoesqueleto de artrópodes. As principais fontes comerciais de quitina são caranguejo e camarão, que são abundantemente fornecidos como produtos residuais da indústria de frutos do mar (Jayakumar et al., 2010). A quitina contém grupos hidroxila e $\mathrm{N}$-acetil em sua estrutura, que são capazes de se ligar com alguns íons em meio aquoso (Dotto et al., 2012, 2013, 2015). Com base no melhor de nosso 
conhecimento, não há estudos na literatura sobre a biossorção de ouro a partir de soluções lixiviadas usando quitina como biossorvente.

Este estudo investigou a possibilidade recuperação de ouro de soluções lixiviadas de microprocessadores de computador descartados (MCD) usando biossorção com quitina. A quitina e os MCD foram obtidos e caracterizados. O ouro foi lixiviado a partir de MCD usando tioureia. Em seguida, foram estudadas duas maneiras para a recuperação de ouro a partir das soluções lixiviadas: (1) biossorção e (2) precipitação seguida por biossorção. Para cada rota, a etapa de biossorção foi estudada em detalhe.

\section{MATERIAIS E MÉTODOS}

\subsection{Preparação e caracterização da quitina}

A quitina foi obtida a partir de resíduos de camarão ( $P$. brasiliensis). Os resíduos foram submetidos à desmineralização, desproteinização, desodorização e secagem (Dotto et al., 2013). A quitina foi moída (Wiley 03) e peneirada, sendo utilizado o tamanho de partícula entre 105-125 $\mu \mathrm{m}$.

A quitina foi caracterizada de acordo com o ponto de carga zero $\left(\mathrm{pH}_{\mathrm{ZPC}}\right.$ ) (Hao et al., 2004), grau de desacetilação, espectroscopia de infravermelho (FT-IR) (Prestige, Japão), área da superficial, volume de poro e raio médio de poro médio (RP) (Quantachrome, EUA). O índice de cristalinidade foi determinado por difração de raios-X (Rigaku, Japão). As características texturais foram visualizadas por microscopia eletrônica de varredura (MEV) (Jeol, Japão).

\subsection{Obtenção e caracterização dos pinos de microprocessadores}

Foram utilizados microprocessadores de computador obtidos em vários pontos de coleta na cidade de Santa Maria - RS. Os pinos foram retirados manualmente com o auxílio de um alicate. Depois de coletados, varias marcas e modelos foram separados a fim de testar em solução de água régia qual marca apresenta o maior percentual de ouro nos pinos. Depois de realizados os testes e analisados por espectrometria de absorção atômica, concluiu-se que a marca Intel pentium ${ }^{\circledR}$ foi a que gerou um maior percentual de massa de ouro em seus pinos.

\subsection{Lixiviação de ouro a partir dos microprocessadores}

Os pinos foram lixiviados com tioureia (Ubaldini et al., 1998). Inicialmente, 1,0 g dos pinos de foram colocados em um frasco volumétrico. Logo após, $20 \mathrm{ml}$ de solução de tioureia $\left(50 \mathrm{~g} \mathrm{~L}^{-1}\right)$ e $20 \mathrm{~mL}$ de sulfato férrico $\left(15 \mathrm{~g} \mathrm{~L}^{-1}\right)$ foram adicionados ao frasco. Em seguida foi adicionado $\mathrm{H}_{2} \mathrm{SO}_{4}$ até que a solução atingisse $\mathrm{pH} 1$. Finalmente, $50 \mathrm{~mL}$ de água deionizada foram adicionados para completar o volume do frasco. A solução foi agitada a $100 \mathrm{rpm}$ por $60 \min$ a $25 \pm 2^{\circ} \mathrm{C}$. No final do experimento, as soluções lixiviadas (contendo ouro, reagentes e metais extraídos) foram filtradas (Whatmann $\mathrm{n}^{\circ} 40$ ) e mantidas em um local escuro para uso posterior. As concentrações de ouro e ferro foram determinadas por espectrômetro de absorção atômica em chama (Agilent, EUA).

\subsection{Biossorção de ouro a partir das soluções lixiviadas}

Duas rotas foram utilizadas para o estudo da biossorção de ouro a partir de soluções lixiviadas: (1) biossorção antes da precipitação e (2) biossorção após a precipitação. $\mathrm{O}$ esquema experimental para a biossorção de ouro de soluções lixiviadas é demonstrado na Figura 1.

\subsubsection{Precipitação: A precipitação foi} realizada com intenção de remover outras substâncias na solução e consequentemente melhorar o desempenho da biossorção. A precipitação foi realizada com $\mathrm{NaOH} 5,00$ mol $\mathrm{L}^{-1}$ até $\mathrm{pH}$ 6. Após a adição do mesmo o 
volume total da solução foi duplicado. Ao final da precipitação, o lodo foi removido e a fase líquida foi utilizada para os experimentos de biossorção.

Figura 1 - Esquema experimental

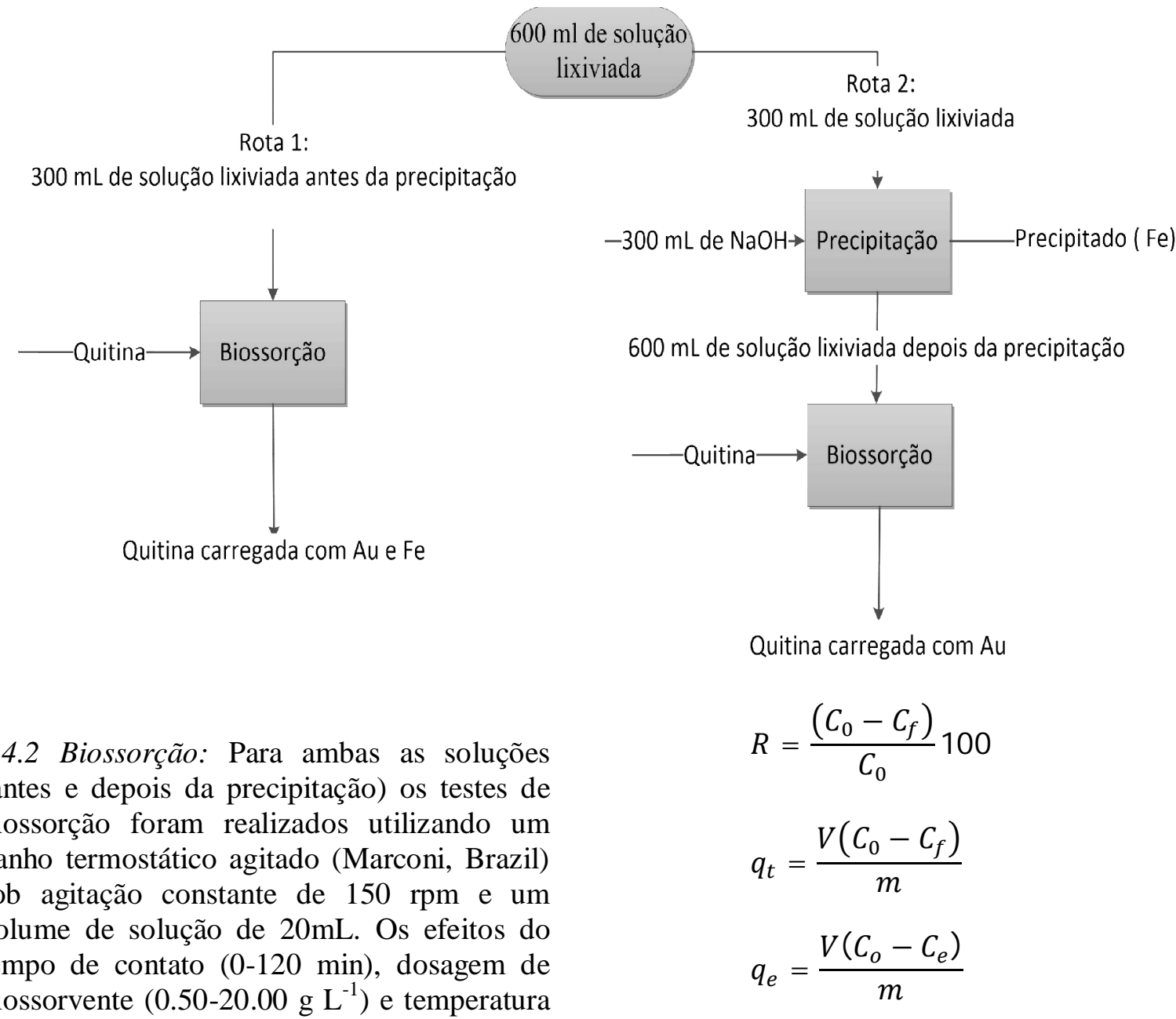

onde, $C_{0}$ é a concentração inicial de ouro em fase líquida $\left(\mathrm{mg} \mathrm{L}^{-1}\right), C_{e}$ é a concentração de ouro no equilíbrio na fase líquida $\left(\mathrm{mg} \mathrm{L}^{-1}\right), C_{f}$ é a concentração final de ouro na fase líquida $\left(\mathrm{mg} \mathrm{L}^{-1}\right), m$ é a massa de biossorvente $(\mathrm{g})$ e $V$ é o volume de solução (L).

\subsubsection{Cinética e equilíbrio de biossorção}

Os dados experimentais da cinética de $q_{e}, \mathrm{mg}^{-1}$ ) foram determinadas através das equações 1,2 e 3 : biossorção de ouro antes pra precipitação e depois da precipitação foram obtidos de 0 a 120 minutos. Estes dados foram interpretados 
a partir das regressões dos modelos de pseudo-primeira ordem (PPO) (eq. 4) (Lagergren, 1898), pseudo-segunda ordem (PSO) (eq. 5) (Ho and McKay, 1998) e de ordem geral (OG) (eq. 6) (Alencar et al, 2012).

$$
\begin{aligned}
& q_{t}=q_{1}\left(1-\exp \left(-k_{1} t\right)\right) \\
& q_{t}=\frac{t}{\left(1 / k_{2} q_{2}^{2}\right)+\left(t / q_{2}\right)} \\
& q_{t} \\
& =q_{n} \\
& -\frac{q_{n}}{\left[k_{n}\left(q_{n}\right)^{n-1} t(n-1)+1\right]^{1 /(n-1)}}
\end{aligned}
$$

Sendo, $k_{1}\left(\min ^{-1}\right), k_{2}\left(\mathrm{~g} \mathrm{mg}^{-1} \min ^{-1}\right)$ e $k_{n}$ $\left(\min ^{-1}\left(\mathrm{~g} \mathrm{mg}^{-1}\right)^{\mathrm{n}-1}\right)$ as constantes da taxa para os modelos: $q_{1}, q_{2}$ e $q_{n}\left(\mathrm{mg} \mathrm{g}^{-1}\right)$ os valores teóricos de capacidade de biossorção e " $n$ " é a ordem da reação de biossorção.

As isotermas de equilíbrio de biossorção foram obtidas a 298, 308, 318 e 328 K. Os modelos de Freundlich (eq. 7) (Freundlich 1906) e BET (eq. 8) (Ebadi et al., 2009) foram ajustados aos dados experimentais.

$$
\begin{aligned}
& q_{e}=K_{f} C_{e}^{1 / n} \\
& q_{e}=\frac{q_{B E T} K_{1} C_{e}}{\left(1-K_{2} C_{e}\right)\left(1-K_{2} C_{e}+K_{1} C_{e}\right)}
\end{aligned}
$$

Onde, $K_{f}$ é a constante de Freundlich ( $(\mathrm{mg} \mathrm{g}$ $\left.\left.{ }^{1}\right)\left(\mathrm{mg} \mathrm{L}^{-1}\right)^{-1 / \mathrm{n}}\right), \quad 1 / \mathrm{n}$ é $\mathrm{o}$ fator de heterogeneidade, $q_{B E T}$ é a capacidade de biossorção em monocamada ( $\left.\mathrm{L} \mathrm{mg}^{-1}\right), K_{1}$ e $K_{2}$ são as constantes de BET ( $\left.\mathrm{L} \mathrm{mg}^{-1}\right)$.

\subsection{Análises do MEVe EDX}

A fim de confirmar os efeitos da precipitação e também da biossorção de ouro, microscopia eletrônica de varredura (MEV) e espectroscopia de raios $\mathrm{X}$ foram utilizados. Para este propósito três amostras de quitina foram analisadas: a quitina antes da biossorção, a quitina biossorvida com ouro antes e após a precipitação.

\section{RESULTADOS E DISCUSSÃO}

\subsection{Características da quitina}

Figura 2 - FT-IR (a), EDX (b) e MEV (c) dfa quitina.
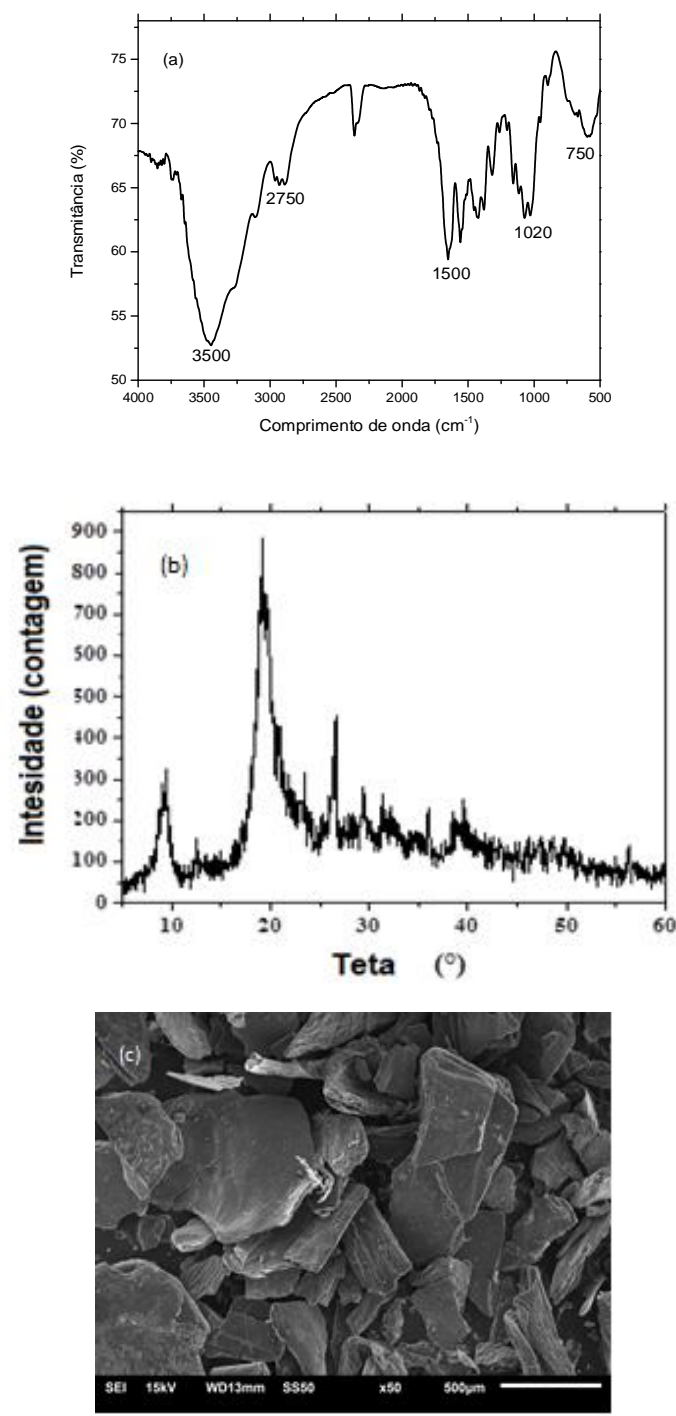

A quitina apresentou ponto de carga zero $\left(\mathrm{pH}_{\mathrm{ZPC}}\right)$ de 5,4. Isto indica que quando o pH da solução é inferior a 5,4 a superfície do adsorvente está carregada positivamente e, a 
superfície está carregada negativamente quando os valores de pH são maiores que 5,4. A área superficial foi de $4,0 \pm 0.2 \mathrm{~m}^{2} \mathrm{~g}^{-1}$, o volume de poros foi de $5,0 \pm 0,2 \mathrm{~mm}^{3} \mathrm{~g}^{-1}$ e o raio médio dos poros foi de $14,8 \pm 0,2 \AA$.

No espectro vibracional (Figura 2 (a)), estão representadas as principais bandas da quitina: 3500 (estiramentos N-H e O-H), 2750 (estiramentos $\mathrm{CH}_{2}$ e $\left.\mathrm{CH}_{3}\right), 1500(\mathrm{C}=\mathrm{O}$ de amida secundária), 1450 (estiramentos de amida II) e 1020 (C-O). Estas bandas são características da quitina, conforme trabalhos anteriores (Dotto et al,,2015). O padrão exibido pelo DRX na Figura 2 (b) mostra que os picos mais relevantes na quitina são quando em $2 \theta$ de aproximadamente $9,5^{\circ} \mathrm{e}$ $20^{\circ}$. Estes dois valores são relativos a estrutura cristalina ortorrômbica da forma- $\alpha$ da quitina (Al-Sagheer et al., 2009). A superfície rígida e não porosa da quitina pode ser confirmada pela Figura 2 (c)

\subsection{Características das soluções lixiviadas}

As características das soluções lixiviadas antes e depois da precipitação são apresentadas na Tabela 1 .

Tabela 1 - Caracterização das soluções lixiviadas.

\begin{tabular}{|c|c|c|}
\hline Característica & $\begin{array}{c}\text { Antes da } \\
\text { precipitação }\end{array}$ & $\begin{array}{c}\text { Depois da } \\
\text { precipitação }\end{array}$ \\
\hline $\begin{array}{l}\text { Volume total } \\
\qquad(\mathrm{mL})\end{array}$ & 300 & 600 \\
\hline $\mathrm{pH}$ & 1,00 & 6,00 \\
\hline $\begin{array}{l}\text { Concentração } \\
\text { de } \mathrm{Au}\left(\mathrm{mg} \mathrm{L}^{-1}\right)\end{array}$ & $202,7 \pm 1,5$ & $97,2 \pm 2,1$ \\
\hline $\begin{array}{c}\text { Quantidade de } \\
\text { Au (mg) }\end{array}$ & 60,8 & 58,3 \\
\hline $\begin{array}{l}\text { Concentração } \\
\text { de Fe }\left(\mathrm{mg} \mathrm{L}^{-1}\right)\end{array}$ & $3001 \pm 2,8$ & $4,2 \pm 0,1$ \\
\hline $\begin{array}{c}\text { Quantidade de } \\
\text { Fe (mg) }\end{array}$ & 900,3 & 2,5 \\
\hline
\end{tabular}

Como pode se observar o valor do $\mathrm{pH}$ foi de 1 para 6 , após a precipitação. Esta elevação de $\mathrm{pH}$ foi favorável para a remoção de $\mathrm{Fe}(99,7 \%)$ e ocorreu uma pequena precipitação de $\mathrm{Au}(4,1 \%)$. Os resultados indicam que a precipitação se mostrou favorável, pois, a maior parte do Fe removida. Este fator se torna importante para o processo, devido diminuir biossorção competitiva entre o Fe e o Au nos sítios disponíveis da quitina.

\subsection{Cinética de biossorção}

As curvas cinéticas para biossorção de ouro a partir de soluções lixiviadas antes e depois da precipitação são apresentadas na Figura 3. As curvas foram ajustadas de acordo com os modelos de pseudo-primeira ordem, pseudo-segunda ordem e de ordem geral. Os parâmetros são apresentados na Tabela 2.

Figura 4 - Curvas cinéticas para biossorção de ouro a partir de soluções lixiviadas antes e após a precipitação.

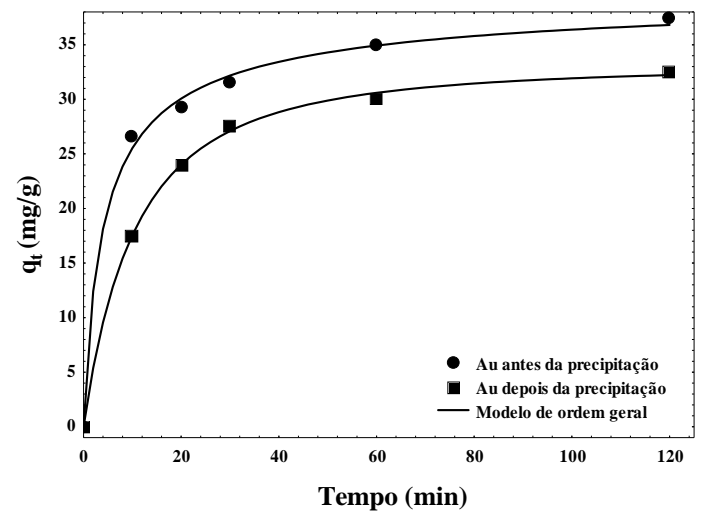

A Figura 4 mostra que $80 \%$ da saturação foi alcançada em 30 min, e após, a taxa de biossorção diminui consideravelmente. Um comportamento semelhante foi encontrado por Park et al (2012).

Baseado nos valores de $\mathrm{R}^{2}, \mathrm{R}_{\text {adj }}^{2} \mathrm{e}$ EMR (Tabela 2) é possível determinar que o modelo que melhor se ajusta aos dados experimentais é o de ordem geral.

É possível observar que o valore de $\mathrm{q}_{\mathrm{n}}$ aumentou $18 \%$ depois da precipitação. Esta mudança no valor pode ser explicada devido às diferenças de concentração de Au. Antes da 
precipitação era duas vezes maior que depois de sua precipitação (Tabela 1). O valor de $h_{o}$ é maior antes da precipitação, o que indicia que o processo de biossorção ocorre mais rápido para está solução. Contudo o valor de $\mathrm{k}_{\mathrm{n}}$ para depois da precipitação é maior que antes da precipitação, isto acontece devido a solução apresentar baixa concentração de Fe $(4,2 \pm 0,1$ $\mathrm{mg} \mathrm{L}^{-1}$ ), facilitando a transferência de $\mathrm{Au}$ da solução para a superfície da quitina.

Tabela 2 - Parâmetros cinéticos estimados para a biossorção de ouro.

\begin{tabular}{ccc}
\hline Modelos & $\begin{array}{c}\text { Antes da } \\
\text { precipita } \\
\text { ção }\end{array}$ & $\begin{array}{c}\text { Depois da } \\
\text { precipitação }\end{array}$ \\
\hline $\mathrm{PPO}$ & 34,7 & 31,26 \\
$\mathrm{q}_{1}\left(\mathrm{mg} \mathrm{g}^{-1}\right)$ & 0,123 & 0,076 \\
$\mathrm{k}_{1}\left(\mathrm{~min}^{-1}\right)$ & 0,974 & 0,995 \\
$\mathrm{R}^{2}$ & 0,967 & 0,994 \\
$\mathrm{R}^{2}$ adj & 5,4 & 2,5 \\
$\mathrm{EMR}(\%)^{\mathrm{PSO}}$ & 37,9 & 35,2 \\
$\mathrm{q}_{2}\left(\mathrm{mg} \mathrm{g}^{-1}\right)$ & 0,0053 & 0,0029 \\
$\mathrm{k}_{2}\left(\mathrm{~g} \mathrm{mg} \mathrm{min}^{-1}\right)$ & 7,6 & 3,7 \\
$\mathrm{~h}_{2}\left(\mathrm{mg} \mathrm{g} \mathrm{min}^{-1}\right)$ & 0,994 & 0,998 \\
$\mathrm{R}^{2}$ & 0,993 & 0,997 \\
$\mathrm{R}^{2}{ }_{\text {adj }}$ & 2,5 & 1,4 \\
$\mathrm{EMR}(\%)^{\mathrm{OG}}$ & & \\
\hline $\mathrm{q}_{\mathrm{n}}\left(\mathrm{mg} \mathrm{g}^{-1}\right)$ & 40,5 & 33,1 \\
$\mathrm{k}_{\mathrm{n}}\left(\mathrm{min}^{-1}\left(\mathrm{~g} \mathrm{mg}^{-1}\right)^{\mathrm{n}-1}\right)$ & $5,78 \mathrm{E}-4$ & $1,41 \mathrm{E}-2$ \\
$\mathrm{~h}_{0}\left(\mathrm{mg} \mathrm{g}^{-1} \mathrm{~min}^{-1}\right)$ & 10,1 & 3,1 \\
$\mathrm{n}$ & 2,64 & 1,54 \\
$\mathrm{R}^{2}$ & 0,997 & 0,999 \\
$\mathrm{R}_{\text {adj }}$ & 0,995 & 0,998 \\
$\mathrm{EMR}(\%)$ & 1,8 & 0,92 \\
\hline
\end{tabular}

\subsection{Equilíbrio de biossorção}

As curvas de equilíbrio de biossorção de ouro estão demonstradas na Figura 4.

As Figuras 4 (a) (antes da precipitação) e 4 (b) (depois da precipitação), mostram que a biossorção de ouro na quitina é representada pela curva tipo S (Giles et al., 1960), indicando forte competição pelos sítios ativos de biossorção. Isto é corroborado, uma vez que, depois da extração do ouro, vários compostos como, Fe, Tioureia e sulfatos permaneceram em solução. Estes compostos provavelmente causam o efeito de biossorção competitiva com o ouro pelos sítios ativos.

Figura 4-Curvas de equilíbrio de biossorção, (a) antes da precipitação, (b) depois da precipitação.
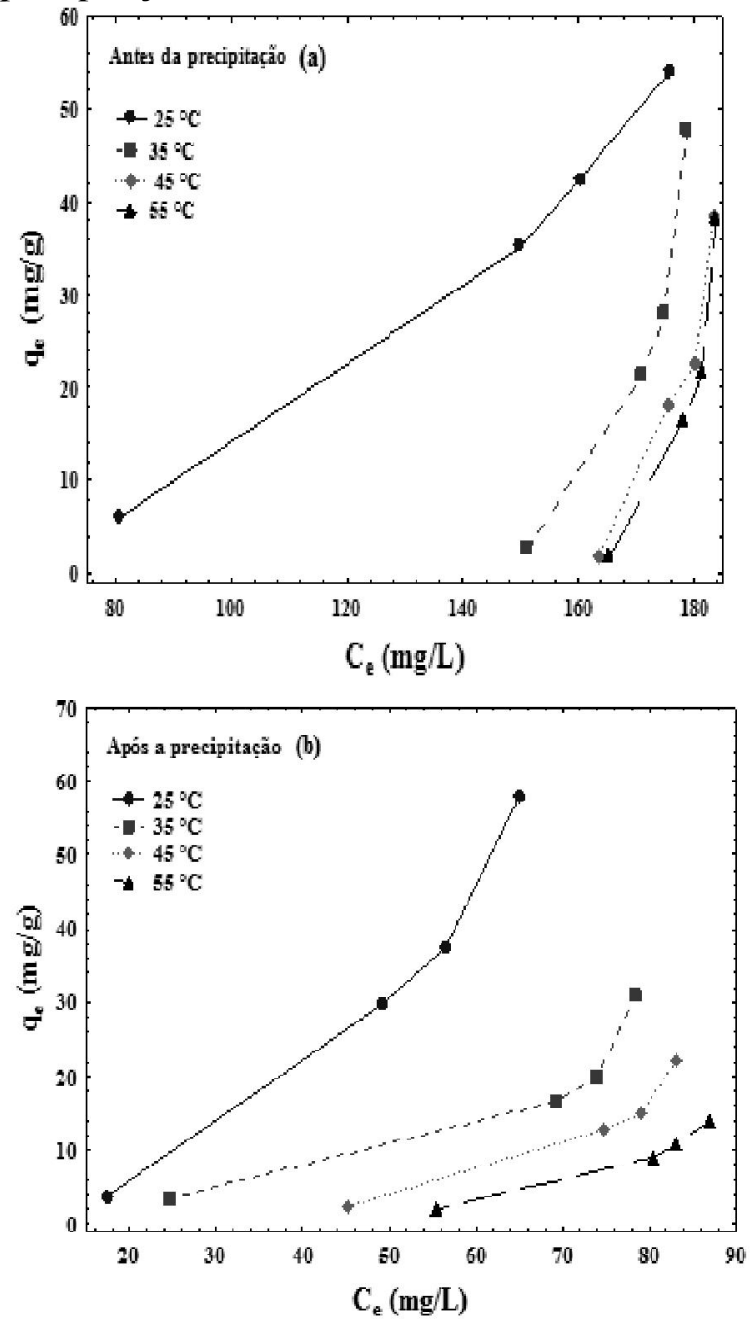

Outro fato visualizado na Figura 4 é que a diminuição da temperatura foi favorável para a biossorção de ouro, a qual alcançou valores máximos em 298K. Comportamento similar em relação a temperatura também foi encontrado por outros autores (Ubaldini et al., 
1998) na biossorção de ouro em cascas de durio zibethinus. Estes verificaram que a capacidade de biossorção decaiu $50 \%$ conforme a temperatura aumentou de $303 \mathrm{~K}$ para $328 \mathrm{~K}$.

Sendo as isotermas do tipo $\mathrm{S}$, os modelos de Freundlich e BET foram ajustados as curvas experimentais com objetivo de obter informações em relação a biossorção. Os parâmetros estimados para os modelos são apresentados na Tabela 3 . Analisando os valores obtidos para $\mathbf{R}^{2}, \mathrm{R}^{2}$ adj $\mathrm{e}$ EMR, pode-se dizer que o modelo de BET foi o que melhor se ajustou aos dados experimentais para biossorção de ouro antes e depois da precipitação. Em geral os valores de

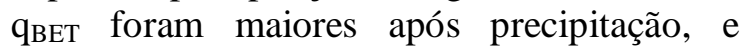
aumentaram com a diminuição da temperatura. Isso mostra que, no equilibro, mais ouro foi biossorvido quando a solução já precipitada foi utilizada. $\mathrm{O}$ mesmo comportamento pode ser verificado para o parâmetro $\mathrm{K}_{2}$, indicando uma maior afinidade entre o ouro e a quitina para as soluções depois de precipitadas a $298 \mathrm{~K}$.

A capacidade máxima de biossorção de ouro obtida foi de $58 \mathrm{mg} \mathrm{g}^{-1}$ para uma temperatura de $298 \mathrm{~K}$, depois da precipitação. Para comparação, Kwak e Yun (2010) obtiveram capacidades de biossorção para o ouro de 50,2 e 86,2 $\mathrm{mg} \mathrm{g}^{-1}$, utilizando respectivamente, Corynebacterium glutamicum in natura e modificado. Park et al., (2012) obtiveram capacidades de biossorção para o ouro de $251,7 \mathrm{mg} \mathrm{g}^{-1}$ utilizando fibras porosas.

Em relação à quantia de ouro removida, a Figura 5 mostra o efeito da dosagem de quitina em relação ao percentual de ouro recuperado da solução lixiviada.

Tabela 3 - Parâmetros de equilíbrio para biossorção de ouro em quitina.

\begin{tabular}{|c|c|c|c|c|c|c|c|c|}
\hline & \multicolumn{4}{|c|}{ Antes da precipitação } & \multicolumn{4}{|c|}{ Depois da precipitação } \\
\hline $\mathrm{T}(\mathrm{K})$ & 298 & 308 & 318 & 328 & 298 & 308 & 318 & 328 \\
\hline \multicolumn{9}{|l|}{ Modelo } \\
\hline \multicolumn{9}{|l|}{ Freundlich } \\
\hline $\mathrm{K}_{\mathrm{f}}\left(\left(\mathrm{mg} \mathrm{g}^{-1}\right)\left(\mathrm{mg} \mathrm{L}^{-1)-1 / n)}\right.\right.$ & $4,4 \mathrm{E}^{-3}$ & $7,0 \mathrm{E}^{-3}$ & $6,6 \mathrm{E}^{-4}$ & $3,7 \mathrm{E}^{-06}$ & $5,9 \mathrm{E}^{-3}$ & $3,3 \mathrm{E}^{-3}$ & $2,0 \mathrm{E}^{-3}$ & $1,6 \mathrm{E}^{-3}$ \\
\hline $\mathrm{n}$ & 0,55 & 0,49 & 0,50 & 0,33 & 0,46 & 0,49 & 0,49 & 0,50 \\
\hline $\operatorname{EMR}(\%)$ & 22,8 & 58,2 & 64,6 & 85,7 & 6,5 & 16,5 & 28,7 & 35,5 \\
\hline $\mathrm{R}^{2}$ & 0,970 & 0,953 & 0,484 & 0,513 & 0,991 & 0,925 & 0,910 & 0,895 \\
\hline $\mathbf{R}_{\text {adj }}^{2}$ & 0,962 & 0,918 & 0,355 & 0,391 & 0,989 & 0,904 & 0,887 & 0,869 \\
\hline \multicolumn{9}{|l|}{ BET } \\
\hline $\mathrm{q}_{\mathrm{BET}}\left(\mathrm{mg} \mathrm{g}^{-1}\right)$ & 9,2 & 6,9 & 1,4 & 0,9 & 37,2 & 3,6 & 7,4 & 8,3 \\
\hline $\mathrm{K}_{1}\left(\mathrm{~L} \mathrm{mg}^{-1}\right)$ & 0,0106 & $8,22 \mathrm{E}^{-03}$ & 0,0080 & 0,0060 & 0,0063 & 0,0682 & 0,0028 & 0,0014 \\
\hline $\mathrm{K}_{2}\left(\mathrm{~L} \mathrm{mg}^{-1}\right)$ & 0,0035 & 0,0052 & 0,0052 & 0,0053 & 0,0099 & 0,0112 & 0,0097 & 0,0090 \\
\hline $\mathrm{K}_{1} \mathrm{q}_{\text {BET }}\left(\mathrm{L} \mathrm{g}^{-1}\right)$ & 0,0984 & 0,0566 & 0,0115 & 0,0051 & 0,2381 & 0,2451 & 0,0212 & 0,0119 \\
\hline EMR (\%) & 5,7 & 9,8 & 8,7 & 9,0 & 9,2 & 2,3 & 3,6 & 4,3 \\
\hline $\mathrm{R}^{2}$ & 0,992 & 0,985 & 0,962 & 0,949 & 0,998 & 0,997 & 0,996 & 0,998 \\
\hline $\mathbf{R}_{\text {adj }}^{2}$ & 0,987 & 0,975 & 0,937 & 0,916 & 0,994 & 0,995 & 0,991 & 0,997 \\
\hline
\end{tabular}

Como pode ser observado na Figura 5, $80 \%$ do ouro foi recuperado da solução (após a precipitação) utilizando de $20 \mathrm{~g} \mathrm{~L}^{-1^{3}}$ de quitina a $298 \mathrm{~K}$ durante $4 \mathrm{~h}$. Baseado nos resultados das Figuras 4 e 5 e da Tabela 3 foi constatado que a etapa de precipitação aplicada antes da biossorção favorece o processo de recuperação de ouro. 
Figura 5 - Remoção de ouro antes e depois da precipitação.

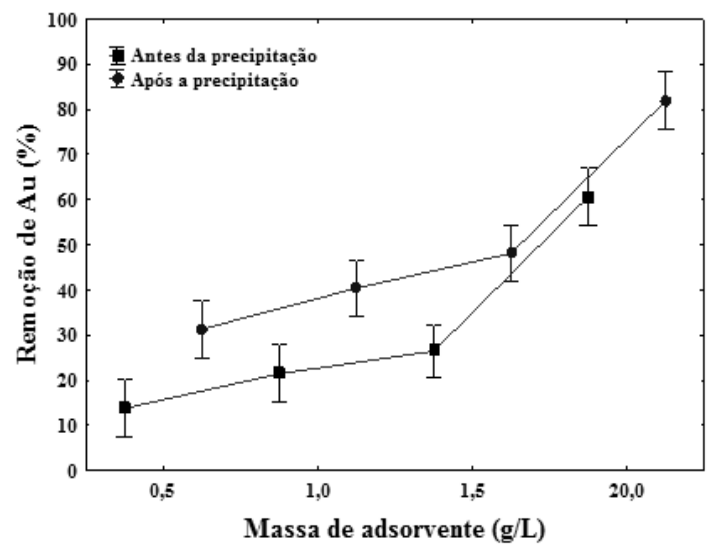

\subsection{Interpretações do MEV e EDX}

As imagens de MEV e espectros de EDX da quitina antes da operação de biossorção (a, b), bioadsorvida com ouro antes da precipitação (rota 1) (c, d) e bioadsorvida com ouro depois da precipitação (rota 2) (e, f) são apresentadas na Figura 6.

Figura 6 - Imagens de MEV e espectros de EDX da quitina.
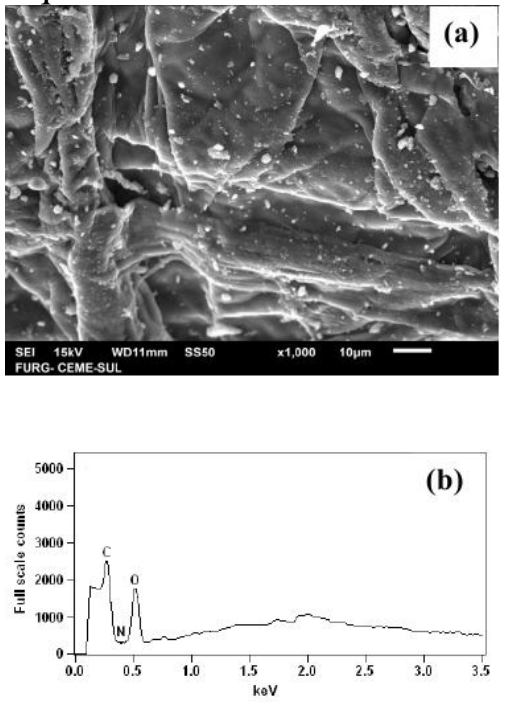
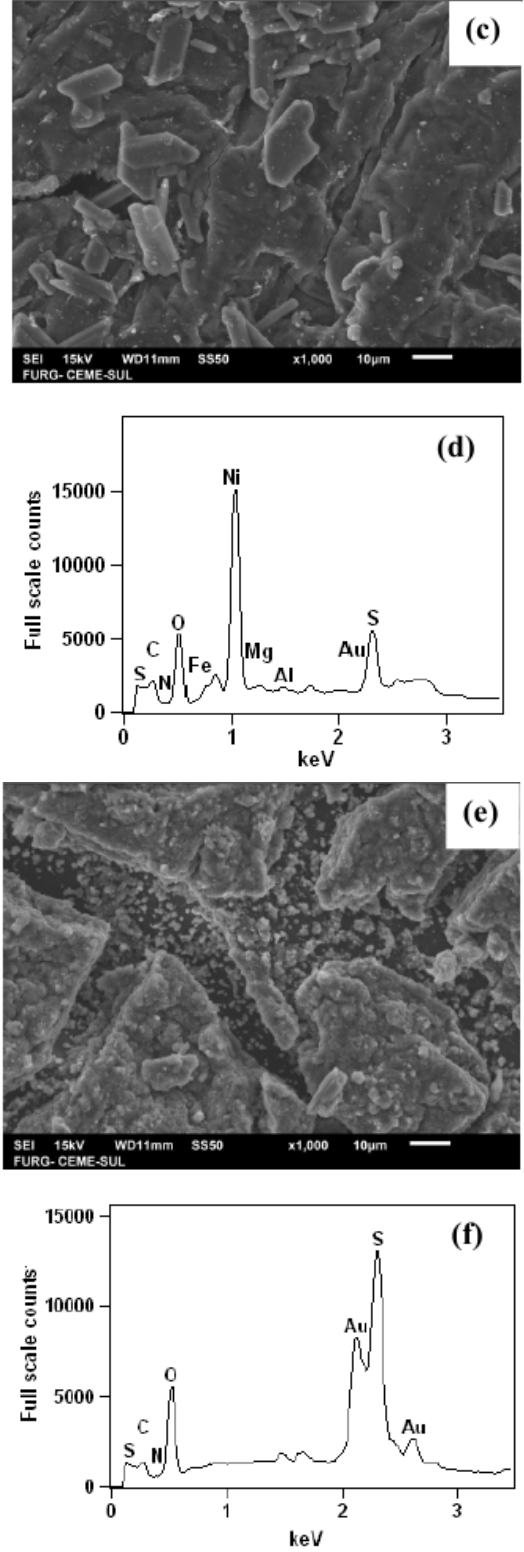

A superfície rígida e não porosa típica da quitina pode ser visualizada na Figura 6 (a). Os elementos comuns presentes na estrutura da quitina são $\mathrm{C} \mathrm{N}$ e $\mathrm{O}$ (Figura 6 (b)). Nas Figuras 6 (c) e 6 (e), pode ser verificado que as características da textura da quitina foram modificadas após o processo de biossorção. Este efeito acontece devido às interações entre a quitina e os componentes previamente lixiviados. Na Figura 6 (d) pode 
ser observado que a quitina foi carregada com diversos elementos, como $\mathrm{S}$ (provavelmente vindo da tioureia, sulfato de ferro ou acido sulfúrico utilizados na operação de extração), $\mathrm{Fe}, \mathrm{Ni}, \mathrm{Mg}, \mathrm{Al}$ e $\mathrm{Au}$ (provenientes da extração). Este fato confirma a forte competição pelos sítios ativos da quitina e corrobora a baixa capacidade de biossorção de ouro na quitina, quando utilizadas soluções sem precipitação. Por outro lado, quando a biossorção foi realizada com soluções após a precipitação, a quitina apresentou apenas $\mathrm{S}$ e $\mathrm{Au}$ na superfície (Figura 7 (f)). Isto revela que diversos componentes foram removidos no estágio de precipitação, desta forma diminuindo a competição por sítios ativos na quitina e por fim melhorando a capacidade de biossorção.

\section{CONCLUSÃO}

Neste estudo, o ouro foi lixiviado de microprocessadores de computador descartados (MCD) e recuperado usando biossorção com quitina. A biossorção foi estudada utilizando as soluções lixiviadas antes e depois da precipitação. Para ambas as soluções, o modelo de ordem geral foi adequado para representar os dados cinéticos. Além disso, os dados de equilíbrio foram bem representados pelo modelo de BET. Os maiores valores de capacidade de biossorção e recuperação de ouro foram encontrados quando se utilizou a solução depois da precipitação. Os resultados de MEV e EDS confirmaram que alguns compostos foram removidos a partir da solução lixiviada no passo de precipitação. Em seguida, concluiuse que a precipitação seguida por biossorção foi mais eficiente para a recuperação de ouro. Usando esta rota, a capacidade de biossorção foi de $58 \mathrm{mg} \mathrm{g}^{-1}$ e cerca de $80 \%$ do ouro foi recuperado a partir da solução.

$1 / \mathrm{n}$

NOMENCLATURA

Fator de

heterogeneidade
$\mathrm{C}_{\mathrm{e}} \quad$ Concentração de ouro no equilíbrio na fase líquida

$\mathrm{C}_{\mathrm{f}} \quad$ Concentração final de ouro na fase líquida

$\mathrm{C}_{\mathrm{o}} \quad$ Concentração inicial de ouro

$\mathrm{K}_{1} \quad$ Constante do modelo de BET

$\mathrm{k}_{1} \quad$ Parâmetro do modelo de PPO

$\mathrm{K}_{2}$ Constante do modelo de BET

$\mathrm{k}_{2} \quad$ Parâmetro do modelo de PSO

$\mathrm{K}_{\mathrm{f}} \quad$ Constante de Freundlich

$\mathrm{k}_{\mathrm{n}} \quad$ Parâmetro do modelo de OG

m Massa de adsorvente

n Ordem da reação de biossorção

$\mathrm{q}_{1} \quad$ Capacidade de biossorção para PPO

$\mathrm{q}_{2} \quad$ Capacidade de biossorção para PSO

qBET Capacidade de biossorção de BET

$\mathrm{q}_{\mathrm{e}} \quad$ Capacidade de equilíbrio de biossorção

$\mathrm{q}_{\mathrm{n}} \quad$ Capacidade de biossorção para OG

Capacidade de biossorção no tempo
$\mathrm{R}$ Percentual de recuperação de ouro

V Volume da solução

\section{REFERÊNCIAS}

ALENCAR, W. S. LIMA, E. C.; ROYER, B. ; SANTOS, B. D. ; CALVETE, T. ; SILVA, 


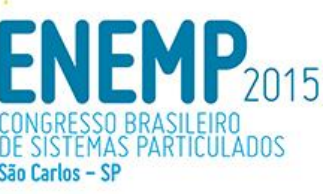

E. A.; ALVES, C. N.; Sep. Sci. Technol. 2012, 47, 513.

AL-SAGHEER, F. A. AL-SUHHAYER, M. A.; MUSLIN, S.; ELSABEE, M. Z. Carbohydr. Polym. 2009, 77, 410.

BERTUOL, D. A.; CALGARO C. O. ; TANABE, E. H. Acid and Alkaline Leaching, in: H. M. Veit and A. Moura Bernardes (Eds.), Electronic Waste, Topics in Mining, Metallurgy and Materials Engineering. Springer International publishing, Switzerland, 2015.

DOTTO, G. L. SANTOS, J. M. N. RODRIGUES, R. ROSA,; PAVAN, F. A.; LIMA.; C. LIMA, J. Colloid Interface Sci. 2015, 446, 133.

DOTTO, G. L. VIEIRA, M. L. G.; L. A. A Pinto, Ind. Eng. Chem. Res. 2012, 51, 6862.

DOTTO, G. L.; ROSA, G. S. ; MORAES, M. A.; WESKA, R. F. ; PINTO, L. A. A., $J$. Environ. Chem. Eng. 2013, 1, 50.

EBADI, A.; SOLTAN, M. J. S.; KHUDEV, A. Adsorption 2009, 15, 65.

FREUNDLICH, H.. Z. Physic. Chem. A57, p. 358-471, 1906.

GILES, C. H. MACEWAN, T. H.; NAKHWA, S. N.; SMITH, D. J. Chem. Soc. 1960, 1, 3973.

HO, Y. S. MCKAY, G. Proc. Safety Environ. Protec. 1998, 76, 332.

HONG, J.; SHI, W.; WANG, Y.; CHEN, W. .; LI, X. Waste Manage. 2015, 38, 357.

JAYAKUMAR, R. PRABARAN, M. ; NAIR, S. V. ; TAMURA, H. Tamura, Biotechnol. Adv. 2010, 28, 142.
KIDDE, P.; NAIDU, R. ; WONG, M. H. Waste Manage. 2013, 33, 1237.

KWAK, I. S.; YUN, Y. S. Bioresour Technol. 2010, $101,8587$.

LAGERGREN, S. Kung. Svenska Vetenskap. 1898, 24,1 .

PARK, S. I. ; KWAK, I. S. ; BAE, M. A. ; MAO, J. ; WON, S. W. ; HAN, D. H. ; CHUNG, Y. S. Yun, Bioresour Technol. 2012, 104, 208.

UBALDINI, S.; FORNARI, R. M.; ABBRUZZESE, C. Hydrometallurgy., v.48, p. 113-124, 1998.

UNGUREANU, G.; SANTOS, S.; BOAVENTURA, R.; BOTELHO, C. J. Environ. Manage. 2015, 151, 326.

VIDYADHAR, A.; CHALAVADI, G. ; DAS, A. J. Environ. Manage. 2013, 118, 49.

WANG, R.; XU, Z. Waste Manage. 2014, 34, 1455.

\section{AGRADECIMENTOS}

Os autores agradecem a CAPES e o CNPq pelo apoio finaceiro. 